\title{
Non-computable Models of Certain First Order Theories
}

\author{
Gábor Sági \\ Alfréd Rényi Institute of Mathematics, \\ Hungarian Academy of Sciences, \\ Reáltanoda u. 13-15, \\ H-1053 Budapest, Hungary \\ and \\ Budapest University of Technology and Economics, \\ Department of Algebra, \\ Egry J. u. 1, \\ H-1111 Budapest, Hungary \\ Email: sagi@renyi.hu
}

\author{
Ramón Horváth \\ Instanridge $\mathrm{AB}$, \\ 11145 Stockholm, Sweden, \\ Birger Jarlsgatan 43, \\ Email: haromn@gmail.com
}

\begin{abstract}
Let $\mathcal{D}$ be a complexity class. A countable first order structure is defined to be $\mathcal{D}$-presented iff all of its basic relations and functions are in $\mathcal{D}$. We show, that if $T$ is a first order theory with at least one uncountable Stone space then $T$ has a countable model not isomorphic to any $\mathcal{D}$-presented one. We also show that there is a countable $\aleph_{0}$-categorical structure in a finite language which is not isomorphic to any $\mathcal{D}$-presented structure; in addition, there exists a consistent first order theory in a finite language that does not have $\mathcal{D}$-presented models, at all.

Our proofs utilize model theoretic methods and do not involve any nontrivial recursion theoretic notion or construction.
\end{abstract}

AMS Subject Classification: 03C57, $03 \mathrm{D} 45$. Keywords: Computable structures, complexity classes, $\aleph_{0}$-categorical structures, oligomorphic permutation groups.

\section{INTRODUCTION}

Abstract data types (for example, in object oriented programming languages) may be regarded as certain first order structures with countable universe and a set of computable operations and relations in it. Further, very often it is important to implement algorithms capable to perform operations in certain countable first order structures (such as algebraic number fields, rings, countably infinite Boolean Algebras, or groups, etc.). Implementing such algorithms have theoretical limitations: all the operations and relations should be recursive in the algorithm theoretical sense. In this paper we investigate such theoretical limitations, the main results are Theorems 4.1 and 5.13; they will be recalled below in the Intorduction after some technical preparations.

A first order structure $\mathfrak{A}$ is defined to be computable iff its universe is the set of natural numbers and all of its basic relations and functions are recursive (in the computational theoretic sense). More generally, if $\mathcal{D}$ is a complexity class (like the set of recursive, or the set of recursively enumerable relations), then a countable structure $\mathfrak{A}$ is defined to be $\mathcal{D}$-presented iff all of its basic relations and functions belong to $\mathcal{D}$. For a more precise definition we refer to Section II below.

By some classical results of Ershov, Arslanov and others, there are countable orderings, Boolean algebras, etc. which are not isomorphic to any computable structure.

One of the main aims of model theory is to describe all structures in which a given theory (i.e. set of first order formulas) is true. At that level of generality this ambitious aim seems to be untractable. Hence, instead of it, model theorists are trying to characterize those theories which have a structure theorem, that is, whose models can be described in a comprehensive way. Recently, related investigations are very active. Along the results of Morley, Shelah, Lascar, Hrushovski, Cherlin, Pillay and others, it turned out, that theories have a "structure theoretic" hierarchy of complexity: in some cases the possible models are relatively easy to describe, in some other cases this is much more difficult, while in some other cases such a complete "comprehensive" description of 
all models is impossible for theoretical reasons. This hierarchy is not as exactly defined as the computational theoretic one. In order to make it more precise, one can "measure the structure theoretic complexity" of a first order theory $T$ by the number of pairwise non-isomorphic models of $T$ of a given cardinality, or by different degrees of stability, i.e. by the size of the Stone spaces of the theory (for the definition of Stone spaces we refer to Section II below). It turned out, that the elements of the above list are closely related to each other. Somewhat roughly, but more concretely, $\aleph_{0}$ categorical theories (which are the simplest ones from structure theoretic point of view) have small (finite) Stone spaces, while the Stone spaces of unstable theories are of large (uncountable) cardinality; if a theory has an uncountable Stone space over the empty set, then it has uncountably many pairwise non-isomorphic countable models.

Some resluts relating model theoretic and recursion theoretic complexity have already been established. For example, the authors in [9] and in [8] deal with countable, computable models of uncountably categorical theories. For further related results we refer the reader to [10] and to [6]; they also contain a rather complete list of references. For more recent related investigations we refer to [5] and [1].

Some of the known results are "positive", that is, they guarantee the existence of a computable model. Negative results state, that certain models do not have recursively presented isomorphic copies (but do not exclude the existence of a $\mathcal{D}$ presented isomorphic copy, where $\mathcal{D}$ is a higher complexity class - like the class of arithmetical relations, for example).

In this paper we are also trying to compare structure theoretic and computational theoretic complexities of first order theories, and we are interested in "strong negative results", that is, our aim is to prove that certain theories have countable models having highly non-computable isomorphic copies, only. In more detail, the main results of the paper are Theorems 4.1 and 5.13; to claim them, let $\mathcal{D}$ be an arbitrary complexity class.

In Theorem 4.1 we show that if a theory $T$ is complicated in the model theoretic sense (i.e. at least one of it's Stone spaces is uncountable) then $T$ has a "complicated" countable model. More precisely, $T$ has a countable model which is not isomorphic to any $\mathcal{D}$-presented structure.

In Theorem 5.13 we show, that there exist a finite first order language and a theory $T$ in it, which is as simple as possible from the model theoretic sense (namely, $T$ is consistent and $\aleph_{0}$-categorical, hence all of its Stone-spaces are finite), but the unique countable model of $T$ does not have a $\mathcal{D}$ presented isomorphic copy.

To prove theorem 5.13, it would be enough for us to construct uncountably many pairwise "essentially different" oligomorphic permutation groups on $\omega$. For completeness, we show in theorem 5.11 that there are $2^{\aleph_{0}}$ many such groups.

There is another related interesting question which we are able to answer. Let $\mathcal{D}$ be a fixed complexity class. As we mentioned, a structure is used to be considered "complicated" from the recursion theoretic point of view, if it is not isomorphic to any $\mathcal{D}$-presented one. It seems to be also natural to replace "isomorphic" with "elementarily equivalent" in the above sentence and to ask whether a first order structure is elementarily equivalent with a $\mathcal{D}$-presented one. For example, if $\mathcal{D}$ is the class of recursive relations and a structure $\mathfrak{A}$ is elementarily equivalent with a $\mathcal{D}$-presented one, then (some fragments of) the theory of $\mathfrak{A}$ may be algorithmically decided. According to our knowledge, there are no previous investigations for structures elementarily equivalent to $\mathcal{D}$-presented ones. In Theorem 5.13 we also show that

there is a finite language $L$ and an $L$ structure $\mathfrak{A}$ which is not elementarily equivalent to any $\mathcal{D}$-presented structure (that is, the first order theory of $\mathfrak{A}$ does not have $\mathcal{D}$-presented models, at all).

Our proofs utilize model theoretic methods and do not involve any nontrivial recursion theoretic notion or construction. Since we believe, that the presented results may be interesting both for model theorists and for recursion theorists, we will present more details than as usual. Particularly, for the readers convenience, sometimes we will include known proofs in the present paper.

The structure of the paper is as follows. At the end of the present section we are summing up our system of notation. In Section II we recall the recursion theoretic and model theoretic notions we will dealing with. In Section III we present some observations which we will use in later sections. In Section IV we prove Theorem 4.1: if a theory has an uncountable Stone space, then it has a countable model which does not have a $\mathcal{D}$-presented isomorphic copy. In Section $\mathrm{V}$ we are dealing with $\aleph_{0}$-categorical structures. In Theorem 5.13 we show that there is a finite language $L$ and an $\aleph_{0}$-categorical $L$-structure which does not have a $\mathcal{D}$-presented isomorphic 
copy; in addition, there is a consistent first order theory in $L$ which does not have a $\mathcal{D}$-presented model. In Theorem 5.11 we also show that there are $2^{\aleph_{0}}$ many closed oligomorphic permutation groups on $\omega$ having pairwise different orbit sequences. Finally, in Section VI we present a question that remained open.

\section{Notation}

Our notation is mostly standard, but the following list may help.

Throughout $\omega$ denotes the set of natural numbers and for every $n \in \omega$ we have $n=\{0,1, \ldots, n-1\}$. Let $A$ and $B$ be sets. Then ${ }^{A} B$ denotes the set of functions from $A$ to $B,|A|$ denotes the cardinality of $A,[A]^{<\omega}$ denotes the set of finite subsets of $A$ and if $\kappa$ is an ordinal then ${ }^{<\kappa} A$ denotes the set of $A$-termed sequences of length smaller than $\kappa$. If $s$ and $t$ are sequences, then $s \frown t$ denotes their concatenation.

Throughout we use function composition in such a way that the rightmost factor acts first. That is, for functions $f, g$ we define $f \circ g(x)=f(g(x))$. Structures are denoted by gothic letters, like $\mathfrak{A}$ or $\mathfrak{B}$; the universe of a given structure will be denoted by the same latin letter $A$ or $B$, respectively. The automorphism group of the structure $\mathfrak{A}$ will be denoted by $\operatorname{Aut}(\mathfrak{A})$.

\section{COMPLEXITY}

We start by recalling the notion of complexity classes.

Definition 2.1: Consider two relations on $\omega$, say $R_{1} \subset \omega^{n}$, and $R_{2} \subset \omega^{m}$. We say that $R_{1}$ has a reduction to $R_{2}$, in symbols $R_{1} \prec R_{2}$, iff there exsists a recursive algorithm or map, say $M$, such that $w \in R_{1} \Leftrightarrow M(w) \in R_{2}$. We say that $R_{1}$ is recursive relative to $R_{2}$.

This definition means that $R_{2}$ is at least as a hard problem as $R_{1}$. If we have an algorithm solving the membership problem for $R_{2}$ then, up to recursivity, it may also be utilized to solve the membership problem for $R_{1}$ as well. It is easy to see that $\prec$ is a reflexive and transitive relation. Consequently - as it is well known - the relation $\prec$ determines an equivalence relation $\sim$ via the stipulation:

$$
R_{1} \sim R_{2} \text { iff } R_{1} \prec R_{2} \text { and } R_{2} \prec R_{1} \text {. }
$$

Definition 2.2: By a complexity class we mean an equivalence class of $\sim$ containing relations of arbitrary arity on $\omega$.

These kind of complexity classes are called Turing degrees or degrees of unsolvability (see [2]). We denote them by calligraphic letters like $\mathcal{D}$. Typical examples for complexity classes are

- the set of recursive relations;

- the set of recursively enumerable relations;

- the set of arithmetical relations.

(If we had used a reduction with more restrictions we would have obtained a more detailed classification such as Karp-classes, for example.)

We recall the following well known facts from [2].

Lemma 2.3: (1) A complexity class is always countable; (2) The set of all complexity classes has cardinality $2^{\aleph_{0}}$; (3) Comlexity classes are partially ordered by the induced ordering of $\prec$; (4) A set of complexity classes has an upper bound (under $\prec)$ iff it is countable.

Now we recall some model theoretic notions.

Let $\kappa$ be a cardinal and $T$ a set of first order formulas. Then $T$ is defined to be $\kappa$-categorical iff up to isomorphism, $T$ has a unique $\kappa$-sized model. A structure is $\kappa$-categorical iff its theory is $\kappa$-categorical.

Let $\mathfrak{A}$ be a structure, $X \subseteq A$ and $n \in \omega$. Then the $n^{\text {th }}$ Stone space $S_{n}^{\mathfrak{A}}(X)$ of $\mathfrak{A}$ over $X$ is the topological dual space of the Boolean algebra of $n$ ary relations definable in $\mathfrak{A}$ with parameters from $X$. Elements of $S_{n}^{\mathfrak{A}}(X)$ are called types (more precisely, they are called $n$-types of $\mathfrak{A}$ over $X$ ). Similarly, if $T$ is a theory (i.e., $T$ is a set of first order formulas) then the $n^{\text {th }}$ Stone space $S_{n}(T)$ of $T$ is the topological dual space of the Boolean algebra of $n$-ary relations definable in $T$; elements of $S_{n}(T)$ are also called types of $T$. For further details we refer to [7].

\section{PRELIMINARY OBSERVATIONS}

Let $L$ be a finite first order language and $\mathcal{D}$ be a complexity class. We say, that an $L$-structure $\mathfrak{A}$ has a $\mathcal{D}$-presentation, iff there is a structure $\mathfrak{B}=$ $\left\langle\omega, R_{i}^{\mathcal{B}}\right\rangle_{i \in L}$ such that $\mathfrak{A}$ and $\mathfrak{B}$ are isomorphic and for every $i \in L, R_{i}^{\mathfrak{B}}$ (as a subset of a direct power of $\omega$ ) is in $\mathcal{D}$. In this case we also say, that $\mathfrak{B}$ is $\mathcal{D}$-presented.

Throughout this section $L$ denotes a finite first order language.

Lemma 3.1: Let $\mathcal{D}$ be a complexity class. Suppose $\mathcal{H}$ is an uncountable family of pairwise 
non-isomorphic countable $L$-structures. Then there exists $\mathfrak{A} \in \mathcal{H}$ such that $\mathfrak{A}$ does not have a $\mathcal{D}$ presentation.

Proof: Let $\mathcal{H}_{0}=\{\mathfrak{A} \in \mathcal{H}: \mathfrak{A}$ has a $\mathcal{D}$ presentation $\}$ and for every $\mathfrak{A} \in \mathcal{H}_{0}$ let $\mathcal{D}(\mathfrak{A})$ be a $\mathcal{D}$-presented structure such that $f_{\mathfrak{A}}: A \rightarrow \mathcal{D}(A)$ is an isomorphism between $\mathfrak{A}$ and $\mathcal{D}(\mathfrak{A})$. Observe, that for every distinct $\mathfrak{A}, \mathfrak{B}$ we have $\mathcal{D}(\mathfrak{A}) \neq$ $\mathcal{D}(\mathfrak{B})$, otherwise $\left(f_{\mathfrak{B}}\right)^{-1} \circ f_{\mathfrak{A}}$ would be an isomorphism between $\mathfrak{A}$ and $\mathfrak{B}$. Hence, the function $\alpha: \mathfrak{A} \mapsto \mathcal{D}(\mathfrak{A})$ is injective. In addition, there are only countably many $\mathcal{D}$-presented $L$-structures, so the range of $\alpha$ is countable. It follows, that $\mathcal{H}_{0}$ (which is the domain of $\alpha$ ) is also countable. Consequently, there exists $\mathfrak{A} \in \mathcal{H} \backslash \mathcal{H}_{0}$; this $\mathfrak{A}$ does not have a $\mathcal{D}$-presentation.

Corollary 3.2: (1) There is an ordering on $\omega$ which does not have a computable presentation.

(2) There is a well-ordering on $\omega$ which does not have an arithmetical presentation.

Proof: Since (2) implies (1), it is enough to show (2). Let $\mathcal{H}$ be the set of (isomorphism types of) countable well-orderings and let $\mathcal{D}$ be the set of arithmetical relations on $\omega$. Since $|\mathcal{H}|=\aleph_{1}$, the statement follows from Lemma 3.1.

\section{THEORIES WITH MANY TYPES}

As we mentioned in the Introduction, a theory is used to consider "complicated" from model theoretic point of view iff at least one of its Stone spaces is of uncountable cardinality. In the present section we show that if a theory $T$ has at least one uncountable Stone space (that is, if there is $n \in \omega$ with $\left|S_{n}(T)\right| \geq \aleph_{1}$ ) then $T$ has a countable model which does not have a computable presentation (even does not have a $\mathcal{D}$-presented isomorphic copy, where $\mathcal{D}$ is an arbitrary fixed complexity class).

Theorem 4.1: Let $\mathcal{D}$ be a complexity class and let $T$ be a first order theory in a finite language such that there is an $n \in \omega$ with $\left|S_{n}(T)\right| \geq \aleph_{1}$. Then $T$ has a countable model which is not isomorphic to any $\mathcal{D}$-presented structure.

Proof: We apply transfinite recursion. Suppose that we have a countable set of countable structures $\left\{\mathfrak{A}_{\alpha}: \quad \alpha<\lambda\right\}$ with $\lambda<\aleph_{1}$ such that they are pairwise nonisomorphic and are models of $T$. Each structure can realize only countably many types from $S_{n}(T)$, since a single $n$-tuple realizes a unique $n$-type. Hence, these countably many structres realize countably many types alltogether. Let us choose a type $p \in S_{n}(T)$ which has not been realized yet. It follows, that there is a structure $\mathfrak{B}$ which realizes $p$ and is a model of $T$. The downward Löwenheim-Skolem theorem implies, that there exists a countable elementary substructure $\mathfrak{A}_{\lambda}$ of $\mathfrak{B}$ such that $\mathfrak{A}_{\lambda}$ still realizes $p$. Thus $\mathfrak{A}_{\lambda}$ has the property: $\mathfrak{A}_{\lambda} \vDash T, \mathfrak{A}_{\lambda} \neq \mathfrak{A}_{\alpha}(\alpha<$ $\lambda)$. In this way we construct $\aleph_{1}$ many pairwise nonisomorphic models of $T$. Hence, the statement follows from Lemma 3.1.

Remark 4.2: It is well-known, that $\left|S_{n}(T)\right| \geq$ $\aleph_{1}$ implies $\left|S_{n}(T)\right|=2^{\aleph_{0}}$ (see e.g. theorem 6.3.4 of Hodges [7]).

\section{V. $\aleph_{0}$-CATEGORICAL THEORIES}

As we already mentioned, from structure theoretic point of view, a theory $T$ is as simple as possible, iff it is $\aleph_{0}$-categorical, that is iff $T$ has a unique countable model.

In this section we show, that there exists an $\aleph_{0}$-categorical theory in a finite language whose unique countable model does not have a computable isomorphic copy (that is, altough $T$ is simple from structure theoretic point of view, its unique countable model is still complicated from computatinal theoretic point of view).

In addition, we also show that there is a countable structure which is not elementarily equivalent to any computable (or any $\mathcal{D}$-presented) structures, where $\mathcal{D}$ is a given complexity class.

We recall a well known result of Svenonius, Ryll-Nardzewski and others which establishes a connection between $\aleph_{0}$-categoricity and the size of Stone spaces.

Theorem 5.1: For a theory $T$ the following two conditions are equivalent:

(1) $T$ is $\aleph_{0}$-categorical;

(2) for all $n \in \omega$ we have $\left|S_{n}(T)\right|<\aleph_{0}$.

The proof can be found in practically every monograph on model theory.

To prove our results first we need to recall some further known connections between $\aleph_{0}$-categorical structures and certain permutation groups on $\omega$.

Definition 5.2: A permutation group $\mathcal{G}$ acting on the set $X$ is defined to be closed iff for every permutation $f \in{ }^{X} X$ the following holds:

if for every finite $s \subseteq X$ there is $g_{s} \in G$ such that $\left.f\right|_{s}=\left.g_{s}\right|_{s}$, then $f \in G$. 
Equip $X$ with the discrete topology. Then $\mathcal{G}$ is a closed permutation group iff it is a closed subset of ${ }^{X} X$ in the corresponding product topology. For more details we refer to [7].

Clearly, the automorphism group of a first order structure is closed.

Definition 5.3: A permutation group $\mathcal{G}$ on $X$ is said to be oligomorphic iff for every $n \in \omega$ the group acts on the $n$-tuples in a way that the number of orbits is finite.

If $\mathcal{G}$ is an oligomorphic permutation group on $X$ and $n \in \omega$ then $o_{n}^{\mathcal{G}}$ denotes the number of orbits of $\mathcal{G}$ on the set of $n$-tuples of $X$.

Lemma 5.4: If $\mathcal{G}$ is a closed oligomorphic permutation group on $\omega$ then there exists an $\aleph_{0}$ categorical structure $\mathfrak{A}$ on $\omega$ with $\operatorname{Aut}(\mathfrak{A})=\mathcal{G}$.

Proof: This theorem is well known, a proof can be reconstructed e.g. by combining Theorems 4.1.4 (b) and 7.3.1 of [7].

Lemma 5.5: For any sequence $\left\langle a_{n} \in \omega: n \in\right.$ $\omega)$ there is an oligomorphic group $\mathcal{G}$ for which $o_{n}^{\mathcal{G}}>a_{n}$ for all $n \in \omega$.

Proof: The proof can be found e.g. in Cameron [3] (see Item 3.24 therein).

We say that two oligomorphic permutation groups $\mathcal{F}, \mathcal{G}$ have same orbit sequences iff for every $n \in \omega$ we have $o_{n}^{\mathcal{F}}=o_{n}^{\mathcal{G}}$.

Lemma 5.6: There exist $\aleph_{1}$ many oligomorphic permutation groups on $\omega$ with pairwise different orbit sequences.

Proof: We apply transfinite recursion. Suppose we have $\left\{\mathcal{G}_{\alpha}: \alpha<\beta\right\}$ where $\beta$ is a countable ordinal, and the $\mathcal{G}_{\alpha}$ 's are oligomorphic permutation groups with pairwise different orbit sequences. So, for every $\alpha<\beta$ we have a sequence $s_{\alpha}=\left\langle o_{n}^{\mathcal{G}_{\alpha}}: n \in \omega\right\rangle$. Let $\iota: \omega \rightarrow \beta$ be a surjection. Consider the sequence $\left\langle 1+o_{n}^{\mathcal{G}_{l(n)}}\right.$ : $n \in \omega\rangle$ as an input for Lemma 5.5. This lemma produces a new oligomorphic group $\mathcal{G}_{\beta}$ with at least $1+o_{n}^{\mathcal{G}_{\iota(n)}}$ many orbits on $n$-tuples. Finally we obtain $\left\{\mathcal{G}_{\alpha}: \alpha<\aleph_{1}\right\}$ containing $\aleph_{1}$ many oligomorphic groups with pairwise different orbit sequences.

To prove the main result of the section, Lemma 5.6 would be suitable. For completeness, we show that, in fact, there are $2^{\aleph_{0}}$ many oligomorphic permutation groups on $\omega$ with pairwise different orbit sequences. To do so we need further preparation. We start by recalling two well known lemmas.
Lemma 5.7: If $\mathcal{G}$ is an oligomorphic permutation group on an infinite set then there exists an oligomorphic permutation group on $\omega$ with the same orbit sequence.

A proof can be found in subsection 2.2 of [3]. The idea is to build a first order structure from which the group and its action is first order definable, and then apply the downward LöwenheimSkolem theorem.

Lemma 5.8: Let $\mathcal{G}$ be an oligomorphic permutation group on $\omega$ and let $\overline{\mathcal{G}}$ be its closure (in the topological sense). Then

(1) $\overline{\mathcal{G}}$ is an oligomorphic permutation group;

(2) The orbit sequences of $\mathcal{G}$ and $\overline{\mathcal{G}}$ are the same.

Proof: (1) is easy; (2) is straightforward.

Next, we present two lemmas we need to prove that there are $2^{\aleph_{0}}$ many closed, oligomorphic permutation groups on $\omega$ with pairwise different orbit serquences.

Lemma 5.9: Suppose $F \subseteq{ }^{\omega} \omega$ with $|F|=\aleph_{1}$ and $s \in{ }^{<\omega} \omega$ such that for every $f \in F$ we have $s \subseteq f$. Then there exists $z_{0}, z_{1} \in<\omega \omega$ such that $z_{0} \neq z_{1}, s \subseteq z_{0} \cap z_{1}$ and

$$
\left|\left\{f \in F: z_{0} \subseteq f\right\}\right|=\left|\left\{f \in F: z_{1} \subseteq f\right\}\right|=\aleph_{1} .
$$

Proof: For each $t \in \quad<\omega_{\omega}$ let $F_{t}=\{f \in F: t \subseteq f\}$. First observe, that $F_{t}=\cup_{n \in \omega} F_{t \frown n}$, hence

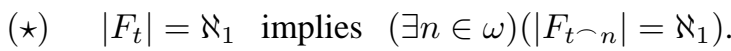

Next, assume, seeking a contradiction, that the statement of the lemma is not true. Let $s_{0}=s$ and suppose $i \in \omega$ and $s_{i}$ has already been defined such that $s \subseteq s_{i}$ and $\left|F_{s_{i}}\right|=\aleph_{1}$. Then by $(\star)$, and by the indirect assumption, there is a unique $n \in \omega$ such that $\left|F_{s_{i} n}\right|=\aleph_{1}$. Let $s_{i+1}=\stackrel{s_{i}}{\neg}$. In this way we defined an increasing sequence $\left\langle s_{i}: i \in \omega\right\rangle$ of finite sequences. Let $f=\cup_{i \in \omega} s_{i}$. Observe, that for any $t \in<\omega \omega$, if $s \frown t \nsubseteq f$ then $\left|F_{s \frown t}\right|<\aleph_{1}$ (because otherwise $z_{0}=s \frown t$ and $z_{1}=\left.f\right|_{|s-t|}$ would contradict to our indirect assumption). Hence

$$
\left|\bigcup_{t \in<\omega \omega, s \frown t \nsubseteq f} F_{s \frown t}\right| \leq \aleph_{0} .
$$

It follows that there exists $g \neq h \in$ $F \backslash \cup_{s \frown t \nsubseteq f} F_{s \frown t}$. But then, for each $n \in \omega$ we have $\left.g\right|_{n},\left.h\right|_{n} \subseteq f$ which implies $\left.g\right|_{n}=h_{n}$ for each $n$. This is impossible, since $g$ and $h$ are different functions. 
Lemma 5.10: Suppose $\left\langle\mathcal{G}_{n}, n \in \omega\right\rangle$ is a sequence of oligomorphic permutation groups such that for every $n \in \omega$ and $k \geq n$ we have $o_{n}^{\mathcal{G}_{n}}=$ $o_{n}^{\mathcal{G}_{k}}$. Then there is an oligomorphic permutation group $\mathcal{G}$ such that for every $n \in \omega$ we have $o_{n}^{\mathcal{G}}=o_{n}^{\mathcal{G}_{n}}$.

Proof: Suppose $\mathcal{G}_{n}$ acts on $X_{n}$, that is, the elements of $G_{n}$ are permutations of the set $X_{n}$. Let $\mathcal{F}$ be a nonprincipal ultrafilter on $\omega$ and let $X$ be the ultraproduct $X=\Pi_{n \in \omega} X_{n} / \mathcal{F}$. Then $\mathcal{G}$ is defined to be the following permutation group on $X$ :

$$
\mathcal{G}=\left\{\left\langle f_{n}: n \in \omega\right\rangle / \mathcal{F}:(\forall n \in \omega)\left(f_{n} \in \mathcal{G}_{n}\right)\right\}
$$

Then the elements of $\mathcal{G}$ are permutations of $X, \mathcal{G}$ is closed under composition and under taking inverses, so $\mathcal{G}$ determines a permutation group. We claim, that $\mathcal{G}$ satisfies the conclusion of the lemma. To see this, let $n \in \omega$ be arbitrary. Then, for every $k \geq n$, one can fix $\left\{s_{i}^{k}: i<o_{n}^{\mathcal{G}_{n}}\right\} \subseteq{ }^{n} X_{k}$ such that, for fixed $k$, the $s_{i}^{k}$,s lying in pairwise different orbits of $\mathcal{G}_{k}$. For each $i<o_{n}^{\mathcal{G}_{n}}$ let $s_{i}=\left\langle s_{i}^{k}: k \in \omega, k \geq n\right\rangle / \mathcal{F}$. It is easy to see, that if $s \in{ }^{n} X$ then there is an $i<o_{n}^{\mathcal{G}_{n}}$ such that $s$ and $s_{i}$ are in the same orbit of $\mathcal{G}$. Hence

$$
\text { (1) } \quad o_{n}^{\mathcal{G}} \leq o_{n}^{\mathcal{G}_{n}}
$$

Conversely, if $i \neq j$ then $s_{i}$ and $s_{j}$ lie in different orbits of $\mathcal{G}$, which implies

$$
\text { (2) } \quad o_{n}^{\mathcal{G}} \geq o_{n}^{\mathcal{G}_{n}}
$$

Combining (1) and (2), the statement follows, as desired.

Theorem 5.11: There are $2^{\aleph_{0}}$ many oligomorphic, closed permutation groups on $\omega$ having pairwise different orbit sequences.

Proof: First note, that $\left|{ }^{\omega} \omega\right|=2^{\aleph_{0}}$, hence there are at most $2^{\aleph_{0}}$ many permutation groups on $\omega$ having pairwise different orbit sequences.

To obtain lower estimation, we show that there is a set $\mathcal{H}^{\prime}$ consisting of $2^{\aleph_{0}}$ many oligomorphic, closed permutation groups with pairwise different orbit sequences. To do so, first we build a tree $T=\left\langle t_{s}, s \in\left\langle\omega_{2}\right\rangle\right.$ where for every $s \in<\omega_{2}$, the elements $t_{s} \in<\omega_{\omega}$ satisfy the following stipulations:

(a) $\left|t_{s}\right| \geq|s|$ and if $s \subseteq s^{\prime}$ then $t_{s} \subseteq t_{s^{\prime}}$;

(b) $s \neq s^{\prime}$ implies $t_{s} \neq t_{s^{\prime}}$;

(c) there are (at least) $\aleph_{1}$ many oligomorphic permutation groups with pairwise different orbit sequences, such that each of these orbit sequences contain $t_{s}$ as an initial segment.

Let $t_{\langle\rangle}=\langle\rangle$. By Lemma 5.6 There exist $\aleph_{1}$ many oligomorphic permutation group with pairwise different orbit sequences, so $(c)$ holds for $t_{\langle\rangle}$. Next, suppose, that $s \in<\omega_{2}$ and $t_{s}$ has already been defined such that (a)-(c) are satisfied. Then, applying Lemma 5.9 we obtain two different extensions $t_{z_{0}}$ and $t_{z_{1}}$ of $t_{s}$ such that $(c)$ remains true if we replace $t_{s}$ in it by $t_{z_{0}}$ or $t_{z_{1}}$. Let $t_{s \frown 0}=t_{z_{0}}$ and let $t_{s \frown 1}=t_{z_{1}}$. In this way (a) and (b) remain true as well, and $T$ can be completely built up.

Now (c) implies, that for every $s \in<\omega_{2}$ there exists an oligomorphic permutation group $\mathcal{G}_{s}$ whose orbit sequence contains $t_{s}$ as an initial segment. In addition, for every $f \in \omega_{2}$ let $\mathcal{G}_{f}$ be the oligomorphic permutation group produced by Lemma 5.10 from the sequence $\left\langle\mathcal{G}_{\left.f\right|_{n}}, n \in \omega\right\rangle$. Then, $t_{\left.f\right|_{n}}$ is an initial segment of the orbit sequence of $\mathcal{G}_{f}$, for each $n \in \omega$. Hence, if $f, g \in{ }^{\omega} 2$ are different, then there exists $n \in \omega$ with $f(n) \neq g(n)$, so by (a) and (b) $\left|t_{\left.f\right|_{n+1}}\right|,\left|t_{\left.g\right|_{n+1}}\right| \geq n+1$ and $t_{\left.f\right|_{n+1}} \neq t_{\left.g\right|_{n+1}}$. In addition, $t_{\left.f\right|_{n+1}}$ and $t_{\left.g\right|_{n+1}}$ are initial segments of the orbit sequences of $\mathcal{G}_{f}$ and $\mathcal{G}_{g}$, respectively. It follows, that $\mathcal{G}_{f}$ and $\mathcal{G}_{g}$ have different orbit sequences. So the set $\mathcal{H}^{\prime}:=\left\{G_{f}, f \in \omega_{2}\right\}$ consists of oligomorphic permutation groups with pairwise different orbit sequences, as desired.

By Lemma 5.7, for every $\mathcal{G}_{f} \in \mathcal{H}^{\prime}$ there exists an oligomorphic permutation group $\mathcal{F}_{f}$ on $\omega$ with the same orbit sequence, by Lemma 5.8 we may assume $\mathcal{F}_{f}$ is closed as well.

Theorem 5.12: There is a finite first order language $L$ in which there are $2^{\aleph_{0}}$ many pairwise non-isomorphic $\aleph_{0}$-categorical structures on $\omega$.

Proof: First we recall some facts from [7]. Suppose $L$ is a language containing a distinguished unary relation symbol $P$ and let $\mathfrak{B}$ be an $L$ structure. We say, that a structure $\mathfrak{A}$ is an induced substructure of $\mathfrak{B}$ by $P$ iff the universe of $\mathfrak{A}$ is $P^{\mathfrak{B}}$ and the definable relations of $\mathfrak{A}$ coincide with the definable relations of $\mathfrak{B}$ restricted to $P$. This determines $\mathfrak{A}$ up to definitional equivalence, only.

By theorem 7.4.8 of Hodges [7], there is a finite language $L$ containing a distinguished unary relation symbol $P$ such that every $\aleph_{0}$-categorical structure $\mathfrak{A}$ (possibly having an infinite language) is an induced substructure of an $\aleph_{0}$-categorical structure $\mathfrak{A}_{L}$ by $P$, where the language of $\mathfrak{A}_{L}$ is $L$.

By Theorem 5.11 there exists a set $\mathcal{H}^{\prime}=\left\{\mathcal{F}_{f}\right.$ : $\left.f \in{ }^{\omega} 2\right\}$ of cardinality $2^{\aleph_{0}}$ containing closed, 
oligomorphic permutation groups on $\omega$ with pairwise different orbit sequences. Lemma 5.4 implies, that for each $\mathcal{F}_{f} \in \mathcal{H}^{\prime}$, there is a countable structure $\mathfrak{A}_{f}$ such that $\operatorname{Aut}\left(\mathfrak{A}_{f}\right)=\mathcal{F}_{f}$. Then $\mathcal{H}^{\prime \prime}=$ $\left\{\mathfrak{A}_{f}: f \in \omega_{2}\right\}$ is a set of pairwise non-isomorphic, countable, $\aleph_{0}$-categorical structures because their automorphism groups are oligomorphic and have pairwise different orbit sequences.

Let $\mathfrak{A}, \mathfrak{B} \in \mathcal{H}^{\prime \prime}$ be arbitrary, but different. Then they have different orbit sequences, hence $\mathfrak{A}_{L}$ cannot be isomorphic to $\mathfrak{B}_{L}$. In other words, the function $\mathfrak{A} \mapsto \mathfrak{A}_{L}$ is injective on $\mathcal{H}^{\prime \prime}$. Let $\mathcal{H}=\left\{\mathfrak{A}_{L}: \mathfrak{A} \in \mathcal{H}^{\prime \prime}\right\}$; clearly $\mathcal{H}$ contains $2^{\aleph_{0}}$ many pairwise non-isomorphic $\aleph_{0}$-categorical $L$ structures, as desired.

Theorem 5.13: Let $\mathcal{D}$ be a complexity class.

(1) There exists an $\aleph_{0}$-categorical structure in a finite language which is not isomorphic to a $\mathcal{D}$ presented structure.

(2) There is a consistent first order theory in a finite language which does not have a $\mathcal{D}$-presented model.

Proof: By Theorem 5.12 there exist a finite language $L$ and a set $\mathcal{H}$ of pairwise non isomorphic, countable $\aleph_{0}$-categorical $L$-structures such that $|\mathcal{H}|=2^{\aleph_{0}} \geq \aleph_{1}$. Now (1) follows from Lemma 3.1.

To show (2), let $\mathfrak{A}$ be a structure satisfying (1) and let $T=T h(\mathfrak{A})$. Since $\mathfrak{A}$ is $\aleph_{0}$-categorical, every countable model of $T$ is isomorphic to $\mathfrak{A}$, hence such a model cannot be $\mathcal{D}$-presented.

Remark 5.14: As we mentioned after Lemma 5.6, Theorem 5.13 may be proved more quickly: by Lemma 5.6 there are (at least) $\aleph_{1}$ many oligomorphic permutation groups on $\omega$ having pairwise different orbit structures. By Lemma 5.8 there are (at least) $\aleph_{1}$ many closed such permutation groups, combining this with Lemma 5.4 and with the technique applied in the proof of Theorem 5.12, we obtain a finite first order language $L$ and $\aleph_{1}$ many pairwise non-isomorphic, countable $\aleph_{0}$ categorical L-structures; applying Lemma 3.1 to this family of structures, we also obtain a proof for Theorem 5.13 .

\section{CONCLUDING REMARKS}

The main results of the paper are Theorems 4.1 and 5.13; they can be summarized as follows. Let $T$ be a first order theory and let $\mathcal{D}$ be a complexity class. According to Theorem 4.1 if $T$ has at least one uncountable Stone space then $T$ has a countable model which is not isomorphic to any $\mathcal{D}$-presented structure. Moreover, according to
Theorem 5.13, there exists a theory $T$ such that all Stone spaces of $T$ are finite, but still, $T$ does not have a $\mathcal{D}$-presented model.

We conclude this work by noting, that the case of theories with countably infinite Stone spaces is still open. From structure theoretic point of view this case has "intermediate complexity". In general, Lemma 3.1 seems unapplicable for them. However, some striking related results can be found in [9].

\section{ACKNOWLEDGMENT}

This work has been supported by Hungarian National Foundation for Scientific Research grant K113047.

\section{REFERENCES}

[1] B. Anderson And B. Csima, Degrees That Are Not Degrees of Categoricity, Notre Dame J. Formal Logic Vol. 57 (2016), no. 3, 389-398.

[2] ED.: J. BARWISE, Handbook of Mathematical Logic, Studies in Logic and the Found. of Math. Vol 90., Elsevier (2006)

[3] P. J. CAmeron, Oligomorphic Permutation Groups, LMS 152., Cambridge University Press, Cambridge (1990).

[4] C. C. Chang, H. J. KeISler, Model Theory, NorthHolland, Amsterdam (1973).

[5] E. B. Fokina, V. Harizanov, A. Melnikov, Computable Model Theory pp. 124-194 in: Turing's Legacy: Developments from Turing's Ideas in Logic, Ed.: R. Downey, Cambridge University Press (2014).

[6] V. S. HARIZANOV, Computability-theoretic complexity of countable structures, Bull. Symbolic Logic 8 (2002), no. 4, 457-477.

[7] W. Hodges, Model theory, Cambridge University Press, (1997)

[8] B. Khoussainov, M. Laskowski, S. Lempr, R. SOLOMON, On the computability-theoretic complexity of trivial, strongly minimal models. Proc. Amer. Math. Soc. 135 (2007), no. 11, 3711-3721.

[9] B. Khoussainov, A. Nies, R. A. Shore, Computable models of theories with few models, Notre Dame J. Formal Logic 38 (1997), no. 2, 165-178.

[10] B. Khoussainov, R. A. SHORE, Effective model theory: the number of models and their complexity, Models and computability (Leeds, 1997), 193-239, London Math. Soc. Lecture Note Ser., 259, Cambridge Univ. Press, Cambridge, 1999.

[11] D. MARKER, Model Theory. An Introduction, GTM 217., Springer-Verlag, New York (2002). 